\title{
A molecular thermometer based on luminescence of copper(II) tetraphenylporphyrin
}

\section{Nozomi Fujino, Musubu Ichikawa*, Toshiki Koyama, and Yoshio Taniguchi}

Department of Functional Polymer Science, Shinshu University

3-15-1 Tokita, Ueda City, Nagano 386-8567, Japan

Corresponding author; Musubu Ichikawa

Postal address; 3-15-1 Tokita, Ueda City, Nagano 386-8567, Japan

e-mail address; musubu@shinshu-u.ac.jp

TEL; +81-(0)268-21-5498, FAX; +81-(0)268-21-5413

\begin{abstract}
The photoluminescence (PL) spectra from solid samples consisting of polystyrene and 5,10,15,20-tetraphenyl-21H,23H-porphine copper(II) (CuTPP) revealed two sharp peaks at 653 and $720 \mathrm{~nm}$, and indicated another broad peak at around $780 \mathrm{~nm}$, which could not be observed in CuTPP solutions. The intensity ratio of the two peaks appearing at $653 \mathrm{~nm}$ and at around $780 \mathrm{~nm}$ demonstrated clear dependence on temperature. This dependence of the PL intensity ratio was observed under the atmosphere and even in water. The findings mean that we were able to utilize PL from CuTPP as a thermometer under various conditions. We measured the temperatures of an organic light-emitting diode (OLED) during operation based on its electroluminescence, where the OLED contained CuTPP as a light emitting dopant, to demonstrate the CuTPP thermometer.
\end{abstract}

Keywords: porphyrin; thermometer; photoluminescence; electroluminescence; organic light-emitting diode 


\section{Introduction}

It is well known that temperature affects numerous chemical, physical, and biological processes and/or activities. Therefore, useful methods of measuring temperature that are suitable for meeting individual objectives are required. Within this context, several useful methods have recently been developed [1-10]. Our laboratory has also demonstrated a method that utilizes the luminescence of a porphyrin derivative (PtOEP: platinum(II) 2,3,7,8,12,13,17,18-octaethyl-21H,23H-porphyrin) as a thermometer to measure the temperature of the emission layer of an organic light-emitting diode (OLED) [11]. A thermometer based on luminescence from a molecule, which we called a molecular thermometer, has many advanteges over other thermometers. For example, this is able to measure the temperature of nano-materials and nano- or micro-devices because the size of the molecules is on an actual nanometer scale. In addition, since the temperature is measured by using the luminescence of the molecular material, we only need a few molecular materials for measurement, which is completely remote and not very time consuming. In other words, if we employ a molecular thermometer based on its luminescence as an OLED light-emitting material, it becomes possible to directrly measure the temperature of the light-emitting layer of the OLED during operation. This is one of the most important advantages of luminescent molecular thermometers, because they make it possible to remotely monitor the temperature of the light-emitting layer, which we cannot attach conventional thermometers to.

Although the molecular thermometer based on PtOEP results in new capabilities as previously mentioned, it has problems in its present state. That is, PtOEP luminescence easily disappears under ambient conditions since the luminescence mechanism for PtOEP is 
phosphorescence, which is greatly influenced by oxygen. Although oxygen obviously affects the fluorescence of molecules, there are many molecules with bright fluorescence around us. Consequently, we believe that we can overcome the obstacle by employing fluorescence-based luminescent molecular thermometers by changing the center metal for porphrin structures. In this paper, we discuss our demonstration of another useful material that works as a molecular thermometer under ambient conditions and even in water.

\section{Experimental}

We prepared two different solid samples: films and fibers, consisting of CuTPP (see Fig. 1) and polystylene (Mw 2.0x10 $)$ from a tetrahydrofuran (THF) solution. Thin-film samples were fabricated by casting the solution on a quartz substrate and then drying it in a vacuum desiccator. Fiber samples were made by wet spinning, where ethanol was used as a poor solvent. In addition, we also fabricated solution samples in various concentrations of CuTPP with chloroform as the solvent. The solution was filtered through a syringe filter whose pore size was $0.2 \mu \mathrm{m}$, and dissolved gases were substituted by argon. The test OLED device (see Fig. 1) consisted of glass coated with indium-tin-oxide (ITO) with a sheet

resistance of $15 \quad \Omega / \quad \square \quad$ used as an anode, $N$, N'-bis(naphthalen-1-yl)-N,N'-bis(phenyl)-benzidine (NPB) as a hole transport layer (30 nm), 5 \% CuTPP doped 4,4'-bis(carbazol-9-yl)biphenyl (CBP) as an emitting layer (40 nm), 2,9-dimethyl-4,7-diphenyl-1,10-phenanthroline (BCP) as a hole blocking layer (15 nm), tris(8-hydroxy-quinolinato)aluminium ( $\left.\mathrm{Alq}_{3}\right)$ as an electron transport layer $(20 \mathrm{~nm})$, and an Al layer $(200 \mathrm{~nm})$ with $\operatorname{LiF}(0.5 \mathrm{~nm})$ as a cathode. The OLED device was fabricated by 
sequentially depositing the layers with thermal evaporation in a vacuum $\left(5.0 \times 10^{-6}\right.$ Torr $)$ onto the ITO glass substrates.

The samples were placed in a constant-temperature oven, and photoluminescence (PL) spectra were recorded with a small spectrograph (Ocean Optics, USB4000) whose spectral sensitivity had been compensated for with a calibrated standard lamp. We used an He-Cd laser as the excitation light source. We also measured time-resolved PL spectra by using a streak scope system (Hamamatsu Photonics, C4334) with a femtosecond pulse laser (Thales, Bright) as the excitation light source, and PL excitation spectra by using a conventional fluorometer (Horiba, FluoroMAX-4P). When we measured the time-resolved PL spectra, we placed a film sample on the cold finger of a cryostat in a vacuum chamber. The OLED was driven by a constant-current power supply (Metromix, Model 5964) and the electroluminescence (EL) spectra were recorded with the same small spectrograph. The temperatures of all samples were measured at the same time with a thermocouple attached directly to the samples. The thermocouple was attached to the opposite surface of the glass on which the sample OLED structure had been prepared. The OLED was encapsulated by a glass cap and fresh desiccant in a glove box filled with pure nitrogen.

\section{Results and Discussion}

Fig. 2 shows the PL spectra of a fiber sample that contains CuTPP in a concentration of $2 \mathrm{wt} \%$ at several different temperatures when we immersed the fiber sample into water. The figure clearly indicates that the PL spectra depend on the temperature. That is, the PL intensities of the broad peak appearing around $780 \mathrm{~nm}$ decreased with increasing temperature. In addition, the same dependence of PL on temperature appeared, when a film 
sample whose doping concentration of CuTPP was the same was placed under the atmosphere. This meant that CuTPP can work as a thermometer under ambient conditions and even in water. The former PtOEP molecular thermometer could only work under inert conditions such as a vacuum due to disappearing PL in the atmosphere.

The inset of Fig. 2 plots the intensity ratio of the two peaks appearing at around 650 $\mathrm{nm}$ and $780 \mathrm{~nm}$ in the fiber sample above as a function of the oven temperature. Note that we determined the intensity of PL at $780 \mathrm{~nm}$ by averaging the intensities within $\pm 1 \mathrm{~nm}$ (there were 15 data within the window) to reduce the influence of the poor signal to noise ratio in the longer-wavelength region. We can see a well-defined linear relationship between the ratio and the temperature from the figure. This clear relationship indicates that everyone can measure the temperature from the intensity ratio of a CuTPP PL spectrum.

We tried to clarify why the PL intensities at around $780 \mathrm{~nm}$ changed depending on the temperature. First, we measured the PL lifetime of CuTPP. The lifetime at 300K of around $780 \mathrm{~nm}(\tau=890 \mathrm{~ns})$ was much longer than that at around 650 and $720 \mathrm{~nm}(\tau=9 \mathrm{~ns})$. This suggests that the longer-wavelength PL component was not the same as the shorter-wavelength ones, and implies that the change in PL intensity depending on temperature might be caused by the non-radiative inactivation processes of the excited state for the broad PL. In general, an excited state with a longer lifetime easily influences inactivation processes. We observed a change in the lifetime in the long-wavelength region depending on temperature (see Fig. 3). The lifetime decreased monotonously with increasing temperature. Actually, as plotted in the inset of the figure, the dependence of the lifetime on temperature could be analyzed by using an Arrhenius-type equation as: 


$$
\frac{1}{\tau(T)}=k_{r}+A \exp \left(-\frac{\Delta E}{k T}\right)
$$

where $k_{r}, A, \Delta E$ and $k$, and $T$ correspond to the radiative transition rate constant, the pre-exponential factor, the activation energy of the non-radiative inactivation process, the Boltzmann constant, and the absolute temperature. We obtained the activation energy for the non-radiative transition process of $0.08 \mathrm{meV}$. Consequently, this indicated that the cause of the decreasing PL intensity at around $780 \mathrm{~nm}$ was the increased nonradiative deactivation that depended on temperature.

We will next explain how the broad peak appears depending on the condition of CuTPP samples, viz., as solutions or in a solid state. Fig. 4 shows the streak scope images of a saturated solution and dilute film of CuTPP at $300 \mathrm{~K}$. The concentration of the solution was $9.4 \times 10^{-3} \mathrm{M}$ and the doping ratio of the film was CuTPP: polystylene $=1: 2 \times 10^{4}$ in weight. No long-lived luminescence was observed in the saturated-solution sample, while a long-lived broad peak appeared in the dilute-film sample. Since the specific gravity of polystylene is $1.05 \mathrm{~g} / \mathrm{cm}^{3}$, the molar concentration of the dilute film was $7.8 \times 10^{-5} \mathrm{M}$. In sum, the saturated solution was more concentrated than the film, but the broad peak only appeared in the film sample. While the field of photochemistry intuitively suggests that the broad peak might originate from the CuTPP excimer, these results probably negate this because no broad peaks appeared even in a dense solution with a concentration of almost $10^{-2} \mathrm{M}$. Note that the sharp peak at $785 \mathrm{~nm}$ is the fundamental wave of the femtosecond laser that was used as the excitation light source.

Fig. 5(a) shows the excitation spectra of the same doped film previously mentioned. The excitation spectra were measured at three observation wavelengths of 660, 720, and 
$785 \mathrm{~nm}$ (we chose the $785 \mathrm{~nm}$ wavelength to reduce the influence of the $720-\mathrm{nm}$ PL because the broad peak had low intensity), and we found differences between the three spectra. The spectra monitored at 660 and $720 \mathrm{~nm}$ were almost the same, but for the spectrum monitored at $785 \mathrm{~nm}$, a new peak appeared and several peaks disappeared. This meant that the luminescence species for the broad peak had to be different from that for the sharp peaks. As described in the previous paragraph, we could not observe any long-lived broad PL in the longer-wavelength region from the solution sample that was more concentrated. In addition, the excitation spectrum of another dilute solution $\left(3.7 \times 10^{-6} \mathrm{M}\right)$ was very similar to that of the dilute film observed at a wavelength of $660 \mathrm{~nm}$ apart from some trivial differences, as plotted in the inset of Fig. 5(b). We concluded from these results that the broad peak was not caused by a CuTPP excimer but probably aggregated CuTPPs that could have been in the solid-state sample, because excimers can be formed by an excited monomer and another ground state monomer, and that is why there was no change in the absorption or excitation spectra from those of the monomer. We are currently planning further experiments to find the origin of the long-lived broad peak.

Finally, we tried to measure the actual temperature of the OLED device during operation at high-current density by using CuTPP as a molecular thermometer. Fig. 6 shows the EL sepctra of the test OLED with CuTPP as the light emitter. The EL spectra closely resembled the PL spectra shown in Fig. 2. The broad peak at around $780 \mathrm{~nm}$ decreases, relatively, with increasing operation-current densities, and this decrease was caused by Joule heat due to the OLED device being driven. We should be able to determine the temperature of an OLED during operation based on its EL spectra by using the relationship 
between temperature and the luminescence-intensity ratio at $650 \mathrm{~nm}$ and $780 \mathrm{~nm}$ as plotted in the inset of Fig. 2. The inset in Fig. 6 plots the temperatures measured with the thermocouple and the intensity ratio of the EL peaks at 650 and $780 \mathrm{~nm}$, where we made two assumptions: (1) the temperature at the lowest current-density operation is the same temperature as that of the thermocouple and (2) the slope of the temperature dependent ratios is constant. This is because the PL intensity ratio of the two peaks did not only depend on temperature but also on CuTPP concentrations. However, we confirmed that the slope of the temperature dependent ratios was independent of the concentration. Consequently, we think that these two assumptions are indeed reasonable. Of course, we know that it would be best to obtain the correct relationship between ambient temperature and the EL intensity ratio of the two peaks from a test OLED that has been placed in a temperature-controlled chamber and driven under very low current-density operation that does not raise temperature. However, we could not do this because the OLED had insufficient EL intensity and durability. Nevertheless, when the OLED was driven at a current density of $450 \mathrm{~mA} / \mathrm{cm}^{2}$, the difference between the two temperatures reached about $44^{\circ} \mathrm{C}$. From basic physical considerations, we understood that the thermocouple's temperature had to be lower than the temperature of the organic layers of the OLED due to the low thermal conductively of glass. In fact, the temperature difference progressively increased when the OLED was driven at dense current densities.

\section{Conclusions}

We have been trying to explore a molecular material for thermometers and found CuTPP to be useful. The luminescence of CuTPP changed depending on the temperature. 
This dependence only appeared in a solid condition, and also appeared even in a very low-concentration sample. This meant that the sample condition is very important to enable CuTPP to work as a molecular thermometer. The thermometer, however, can be used under ambient conditions involving oxygen and moisture. In addition, we measured the temperatures of the OLED device based on the EL of CuTPP during operation. Thus, this molecular thermometer accomplished by using the luminescence of CuTPP should be useful for various purposes in the future.

\section{Acknowledgments}

This work was supported by CLUSTER (the second stage) of the Ministry of Education, Culture, Sports, Science and Technology (MEXT), Japan. 


\section{References}

[1] J. M. Lupton, Appl. Phys. Lett. 81 (2002) 2478.

[2] H. Aizawa, T. Katsumata, S. Komuro, T. Morikawa, H. Ishizawa, and E. Toba, Sens. Actuators, A 126 (2006) 78.

[3] M. Mitsuishi, S. Kikuchi, T. Miyashita and Y. Amao, J. Mater. Chem. 13, (2003) 2875.

[4] K. Iwai, Y. Matsumura, S. Uchiyama, and A. P. de Silva, J. Mater. Chem. 15 (2005) 2796.

[5] S. Katagiri, Y. Hasegawa, Y. Wada and S. Yanagida, Chem. Lett. 33, (2004) 1438.

[6] A. J. Bur, M. G. Vandel, and S. C. Roth, Polym. Eng. Sci. 41 (2001) 1380.

[7] Y. Amao and I. Okura, Bull. Chem. Soc. Jpn. 75 (2002) 389.

[8] Y. Shiraishi, R. Miyamoto, X. Zhang, and T. Hirai, Org. Lett. 9 (2007) 3921.

[9] Y. Shiraishi, R. Miyamoto, T. Hirai, Langmuir 24 (2008) 4273.

[10] N. Chandrasekharan and L. A. Kelly, J. Am. Chem. Soc. 123 (2001) 9898.

[11] T. Sugiyama, H. Tsuji and Y. Furukawa, Chem. Phys. Lett. 453 (2008) 238.

[12] Y. Gao and Y. Bando, Nature 415 (2002) 599.

[13] K. Araki, M. Ichikawa, T. Okino, and Y. Taniguchi, JP Patent Application No. 2006-230024, 28 Aug. 2006. 


\section{Figure captions}

Fig. 1 Chemical structures of materials used and device structure of OLED used.

Fig. 2 Normalized PL spectra of CuTPP doped polystylene fiber in water at different ambient temperatures. Inset plots dependence of PL intensity ratio on temperature of two peaks appearing around $650 \mathrm{~nm}$ and $780 \mathrm{~nm}$.

Fig. 3 Dependence of lifetime on temperature for broad peak appearing at around $780 \mathrm{~nm}$. Inset has Arrhenius-type plot together with fitted curve (solid line).

Fig. 4 Streak scope images of CuTPP sample: (a) Saturated solution and (b) dilute film $\left(7.8 \times 10^{-5} \mathrm{M}\right)$. Solid lines at bottom of each image mean PL spectra constructed by accumulating signals from $0.1 \mu$ s to $1.8 \mu$ s for (a) or from $0.1 \mu$ s to $4.5 \mu$ s for (b).

Fig. 5 (a) Excitation spectra of dilute CuTPP films measured at three observation wavelengths: 660, 720, and $785 \mathrm{~nm}$. (b) Excitation spectra of dilute solution and film observed at wavelength of $660 \mathrm{~nm}$.

Fig. 6 Normalized EL spectra of OLED with CuTPP at different current densities. Inset plots dependence of temperatures on current density measured with thermocouple attached directly to glass substrate surface and calculated by using EL spectra. 


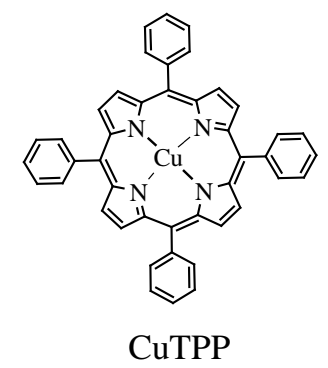

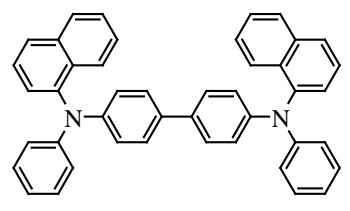

NPB

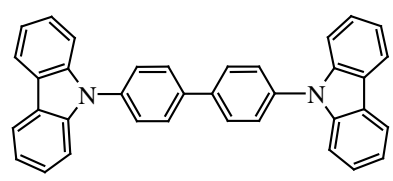

CBP

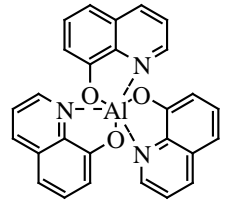

$\mathrm{Alq}_{3}$

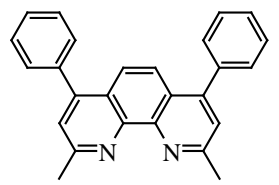

BCP

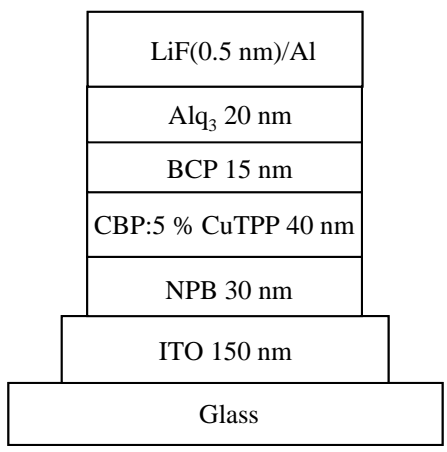

Fig. 1 Chemical structures of materials used and device structure of OLED used. 


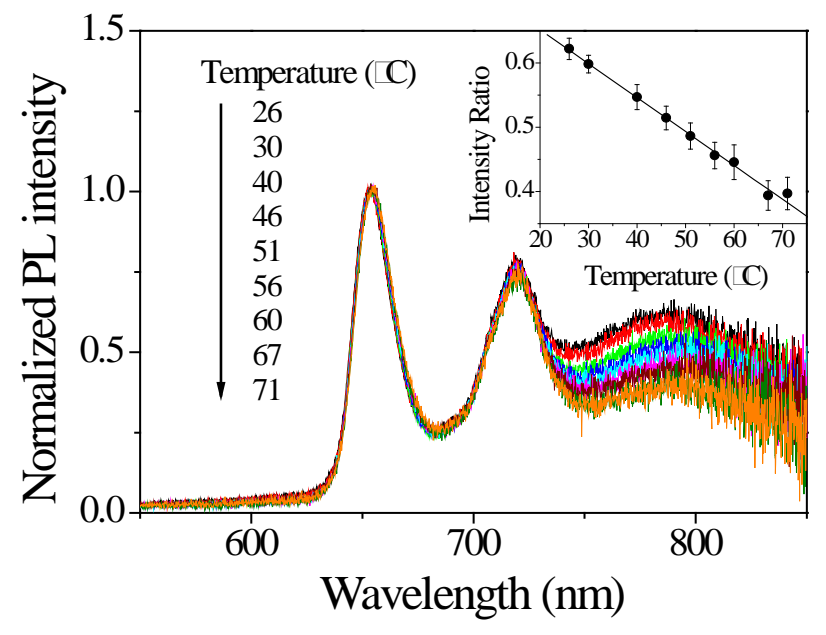

Fig. 2 Normalized PL spectra of CuTPP doped polystylene fiber in water at different ambient temperatures. Inset plots dependence of PL intensity ratio on temperature of two peaks appearing around $650 \mathrm{~nm}$ and $780 \mathrm{~nm}$. 


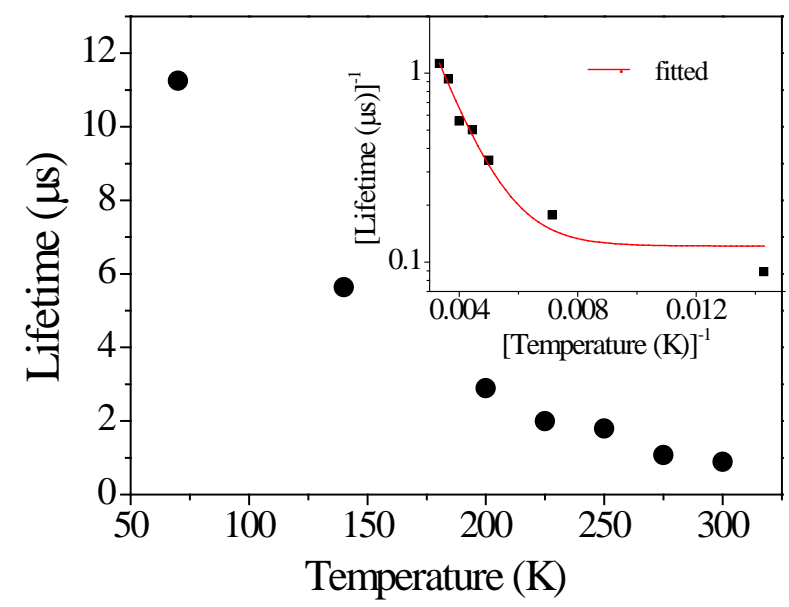

Fig. 3 Dependence of lifetime on temperature for broad peak appearing at around $780 \mathrm{~nm}$. Inset has Arrhenius-type plot together with fitted curve (solid line). 


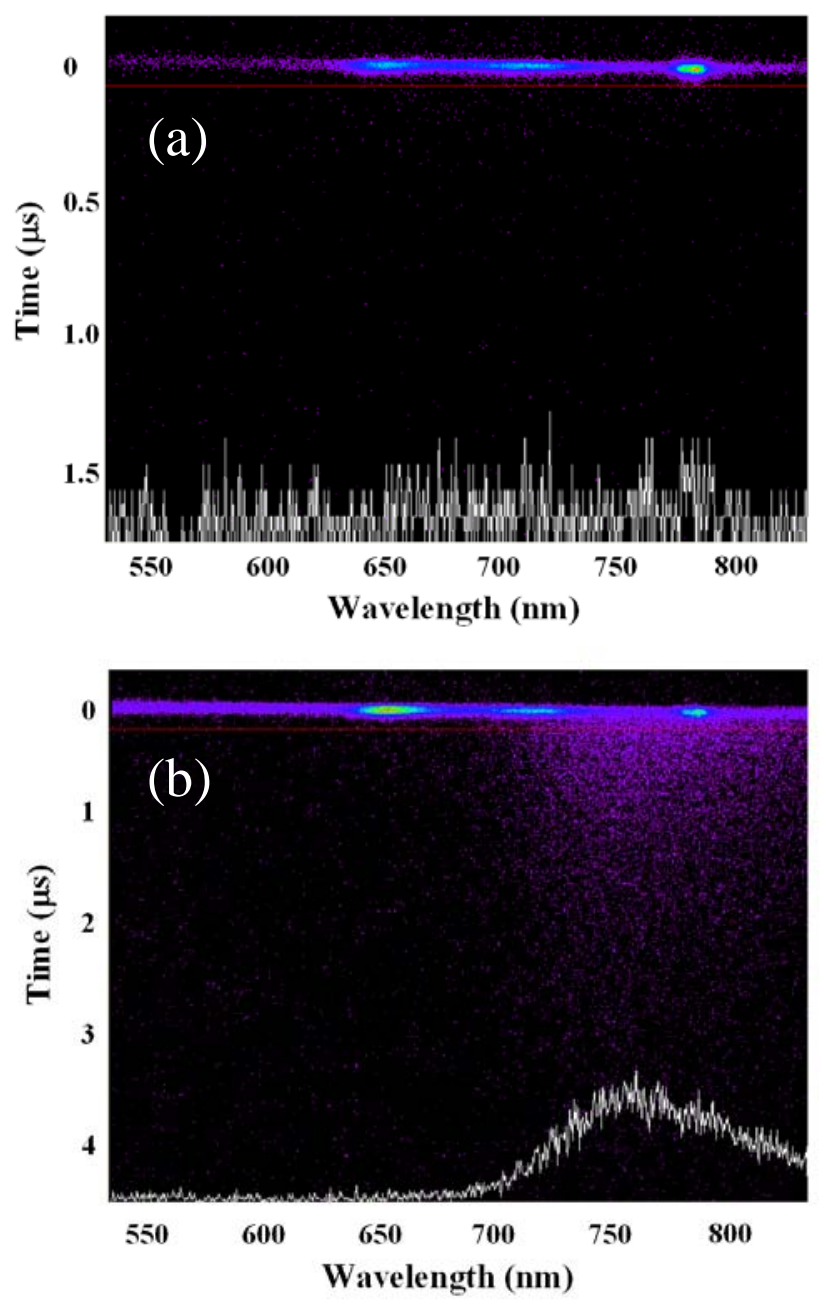

Fig. 4 Streak scope images of CuTPP sample: (a) Saturated solution and (b) dilute film $\left(7.8 \times 10^{-5} \mathrm{M}\right)$. Solid lines at bottom of each image mean PL spectra constructed by accumulating signals from $0.1 \mu$ s to $1.8 \mu$ s for (a) or from $0.1 \mu$ s to $4.5 \mu$ s for (b). 


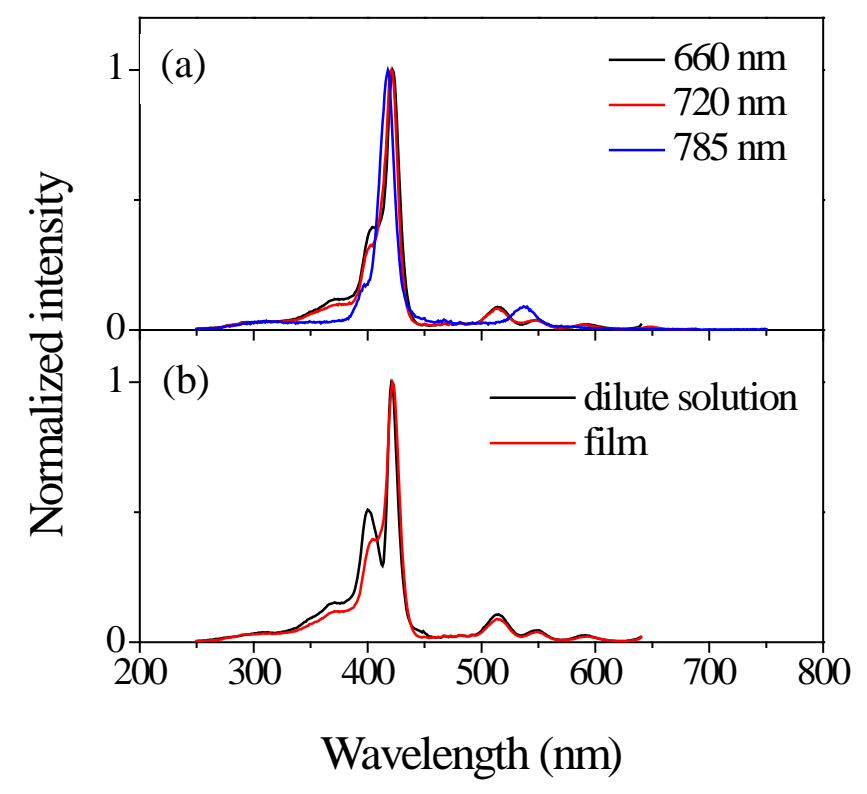

Fig. 5 (a) Excitation spectra of dilute CuTPP films measured at three observation wavelengths: 660, 720, and $785 \mathrm{~nm}$. (b) Excitation spectra of dilute solution and film observed at wavelength of $660 \mathrm{~nm}$. 


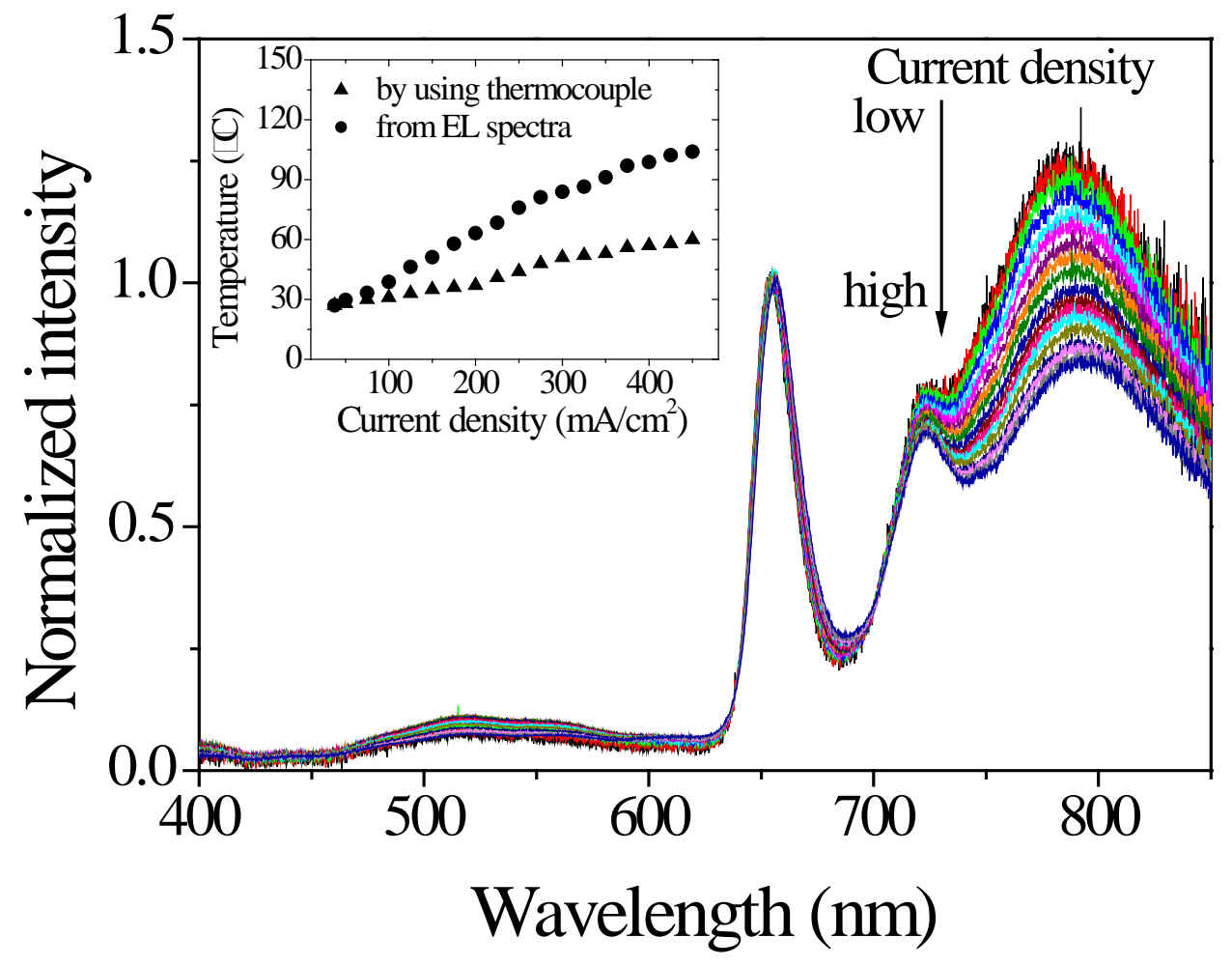

Fig. 6 Normalized EL spectra of OLED with CuTPP at different current densities. Inset plots dependence of temperatures on current density measured with thermocouple attached directly to glass substrate surface and calculated by using EL spectra.. 